\title{
Dielectronic recombination rate in statistical model
}

\author{
A.V. Demura, D.S. Leontyev*', V.S. Lisitsa, and V.A. Shurigyn \\ National Research Center "Kurchatov institute", 123182 Moscow, Russia
}

\begin{abstract}
The dielectronic recombination rate of multielectron ions was calculated by means of the statistical approach. It is based on an idea of collective excitations of atomic electrons with the local plasma frequencies. These frequencies are expressed via the Thomas-Fermi model electron density distribution. The statistical approach provides fast computation of DR rates that are compared with the modern quantum mechanical calculations. The results are important for current studies of thermonuclear plasmas with the tungsten impurities.
\end{abstract}

The dielectronic recombination (DR) plays the important role in the establishing ionization equilibrium of multielectron ions in high temperature plasma. In the modern thermonuclear facilities, tungsten is used for the elements of the discharge camera. Thus, the DR rate calculations of its ions are significant for evaluation of its impurities. The detailed level-bylevel calculations of the DR rate dependence $Q_{d r}(T)$ on plasma temperature $T$ for multielectron ions are a very difficult problem since for complex atoms like tungsten they require to take into account a huge number of atomic states and configurations [1]. The general expression for the DR rate represents itself the sum over branching ratios assembled from the total radiation $W_{R}(i)$ and autoionization $W_{a}(i, n l)$ rates of initial doubly excited states "i" and the partial autoionization rates $W_{a}(\mathrm{i}, n l ; f)$ [1].

$$
\begin{aligned}
Q_{D R}^{p}(T) & =a_{0}^{3}\left(\frac{4 \pi R y}{T}\right)^{3 / 2} \sum_{i n l, f} \frac{g_{i}}{g_{f}} \exp \left(-\frac{\mathrm{h} \omega_{i f}}{T}+\frac{Z_{i}^{2} R y}{n^{2} T}\right) \cdot \frac{W_{a}(\mathrm{i}, n l ; f) \cdot W_{R}(i)}{W_{R}(i)+W_{a}(\mathrm{i}, n l)}, \\
W_{R}(i) & \equiv \sum_{f^{\prime}} W_{R}\left(i, \mathrm{nl} ; f^{\prime}, n l\right), \quad W_{a}(\mathrm{i}, n l) \equiv \sum_{f^{\prime \prime}} W_{a}\left(\mathrm{i}, n l ; f^{\prime \prime}\right),
\end{aligned}
$$

where $a_{0}$ is the Bohr radius, $2 R y$ is the atomic energy unit, $Z_{i}$ is charge of the ion, $g_{i}, g_{f}$ are the statistical weights of the initial and final states of ion core. The autoionization states are described by the sets of quantum numbers of the initial states of the ion core $(i)$ and the ones of external electrons $(n l)$. The set of the quantum numbers $(f)$ describes the final stationary states of the ion core.

In the statistical approach of the multielectron ion is represented by a system of harmonic oscillators with the local plasma frequencies, expressed via the Thomas-Fermi electron density distribution $n_{T F}\left(\mathrm{r} ; Z_{i}, Z\right)$ ( $\mathrm{r}$ is radius vector from nucleus) [2-3]

\footnotetext{
*Corresponding author: leontievdmitiy@gmail.com
} 


$$
\omega_{p}=\left(4 \pi e^{2} n_{T F}\left(\mathrm{r} ; Z_{i}, Z\right) / m\right)^{1 / 2}
$$

where $Z_{i}$ is the ion charge and $Z$ is the charge of ion nucleus, $e, m$ are the charge and mass of electron respectively. This model allows to describe universally different chemical elements. The partial radiation decay rates $W_{R}(i, \mathrm{f})$ could be expressed via the oscillator's strengths $f_{i f}$ (c is the speed of light) [4]

$$
W_{R}(i, \mathrm{f})=2 f_{i f} \omega_{i f} / c^{3}
$$

The partial autoionization decay rate $W_{a}(\mathrm{i}, n l ; f)$ could be represented via the averaged over resonances near threshold the electron excitation cross-section $\sigma_{\mathrm{exc}}(\mathrm{f} ; \mathrm{i}, \mathrm{n} l)$ of the doubly excited state (i,nl) [1, 4-5]

$$
(2 l+1) \mathrm{g}_{f} W_{a}(\mathrm{i}, n l ; f)=Z_{i}^{2} n^{-3} \omega_{i f} g_{i} \sigma_{\text {exc }}(\mathrm{f} ; \mathrm{i}, \mathrm{n} l) / \pi^{2} .
$$

For the simplification of the calculations it is conventional to average $W_{a}(\mathrm{i}, n l ; f)$ over the orbital momentum $l$ of the external electron [1,4-5], that gives with the use of quasiclassical asymptotics the result $W_{a}(\mathrm{i}, n ; \mathrm{f})[5]$

$$
W_{a}(\mathrm{i}, n ; \mathrm{f})=<W_{a}(\mathrm{i}, n l ; f)>_{l} \approx(4 \cdot 1.7 / 3 \pi) \cdot\left(Z_{i}^{2} f_{f i} / n^{5} \omega_{i f}\right) .
$$

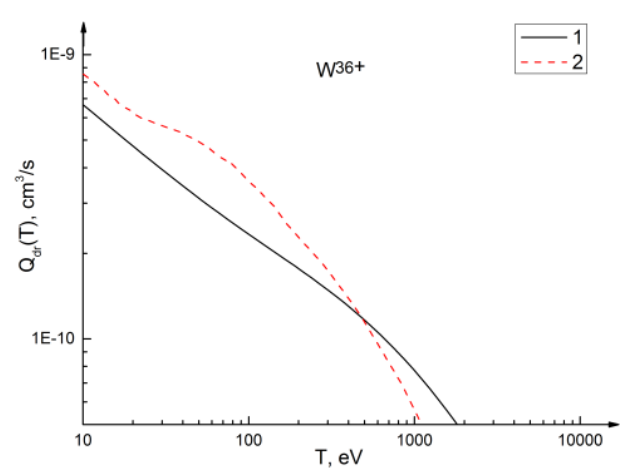

Fig. 1. The total DR rate for the tungsten ion $W^{36+}$ as the function of electron temperature. Curve 1 is the calculation by statistical model; curve 2 is the data of detailed calculation [6].

As in the statistical approach, the oscillator's strengths also are expressed as the functions of electron density distribution the total DR rate is represented at the end as the functional of electron density.

The comparison of the total DR rates for the tungsten ion $\mathrm{W}^{36+}$ dependences versus $\mathrm{T}$, calculated within the statistical approach and by the level-to-level package of codes [6], where finally the DR was evaluated using code FAC [7, 8], is presented in Figure 1. As one can see, there is a good agreement between the statistical model and detailed calculations.

As it could be seen from presented comparison the statistical approach provides the universal description of the DR rates for multielectron ions and allows to facilitate considerably its numerical evaluation.

\section{References}

1. I.I. Sobel'man, L.A. Vainstein, E.A. Yukov, Excitations of Atoms and Broadening of Spectral Lines (Springer, Berlin, 1995)

2. A.V. Demura, M.B. Kadomtsev, V.S. Lisitsa, V.A. Shurygin, Atoms 3, 162 (2015)

3. W. Brandt, S. Lundqvist, Phys. Rev. 139, 612 (1965)

4. W.H. Tucker, R.J. Gould, Astrophys. J. 144, 244 (1966)

5. L.A. Bureyeva, T. Kato, V.S. Lisitsa, Phys. Rev. A 65, 032702 (2002)

6. Z. Wu, C. Dong, Theoretical Study on Electron Impact Excitation and Dielectronic Recombination of Highly Charged Tungsten Ions (IAEA, Vienna, 2014)

7. M.F. Gu, Astrophys. J. 590, 1131 (2003)

8. M.F. Gu, Can. J. Phys. 86, 675 (2008) 